\title{
Bulky spacer groups - A valid strategy to control the coupling of functional molecules to surfaces?
}

\author{
Erik R. McNellis ${ }^{\mathrm{a}}$, Giuseppe Mercurio ${ }^{\mathrm{b}}$, Sebastian Hagen ${ }^{\mathrm{a}, \mathrm{c}}$, Felix \\ Leyssner $^{\mathrm{c}}$, Jörg Meyer ${ }^{\mathrm{a}}$, Serguei Soubatch ${ }^{\mathrm{b}}$, Martin Wolf ${ }^{\mathrm{a}, \mathrm{c}}$, Karsten \\ Reuter $^{\mathrm{a}, \mathrm{d}}$, Petra Tegeder ${ }^{\mathrm{c}}$, F. Stefan Tautz ${ }^{\mathrm{b}, *}$ \\ ${ }^{a}$ Fritz-Haber-Institut der Max-Planck-Gesellschaft, Faradayweg 4-6, 14195 Berlin, \\ Germany \\ ${ }^{b}$ Institute of Bio- and Nanosystems 3, JARA-Fundamentals of Future Information \\ Technology, Forschungszentrum Jülich, 52425 Jülich, Germany \\ ${ }^{c}$ Fachbereich Physik, Freie Universität Berlin, Arnimallee 14, 14195 Berlin, Germany \\ ${ }^{d}$ Department Chemie, Technische Universität München, Lichtenbergstrasse 4, 85747 \\ Garching, Germany
}

\begin{abstract}
We examine a popular decoupling strategy for separating functional molecules from metal surfaces, using the molecular switches azobenzene and TBA as examples. Our combined experimental (Normal Incidence X-ray Standing Wave method) and theoretical (Density Functional Theory) study demonstrates that purely structural arguments to predict the functionality of adsorbed molecules have to be taken with much care, as they may turn out to be deceptive.
\end{abstract}

Keywords: Molecular switches, Isomerism, Azobenzene compounds, NIXSW, DFT

The ability to align purpose-designed molecules on surfaces where they can perform functions such as switching, sensing or catalysis is a key capabil-

\footnotetext{
${ }^{*}$ Corresponding author

Email address: s.tautz@fz-juelich.de (F. Stefan Tautz)
} 
ity in the quest for a molecular nanotechnology. For this purpose, not only the molecules themselves but also the interaction between their functional units and the substrate in question must be tailored carefully. This often requires tuning the coupling to a (metal) surface to protect the molecular functionality against detrimental influences from the substrate (like excited state quenching). A wide-spread strategy to control this coupling is the attachment of bulky spacer groups which are designed to lift the functional unit of the molecule off the surface $[1,2]$. A prototypical example for this approach in the context of molecular switches is provided by recent work addressing the functionality of azobenzene (H5C6-N=N-C6H5), cf. Fig. 1a, at gold surfaces $[3,4,5,6,7,8]$. In solution, this molecule and its derivatives are well-known to switch reversibly between their planar trans- and threedimensional cis-configurations by a photo-induced isomerization [9]. Despite the inertness of the close-packed $\mathrm{Au}(111)$ surface, this switching function is lost in the adsorbed state [3]. A likely cause of this suppression is that the metal quenches the photoexcited state of the molecule by ultrafast electron or energy transfer $[10,11,12,13,14]$. Ostensibly, increasing the distance between the $-\mathrm{N}=\mathrm{N}$ - moiety and the surface restores the switching functionality, which indeed has been found to be the case if tert-butyl groups are attached in the phenyl ring meta positions, cf. Fig. $1 \mathrm{~b}[3,4,5,6,7,8]$.

This finding apparently supports the validity of the concept of geometric decoupling using bulky spacer groups as schematically shown in Fig. 2a and b. In contrast, in this communication we present direct evidence that this widely-used approach is inappropriate and should be replaced by a holistic decoupling strategy which considers the geometric and electronic properties 


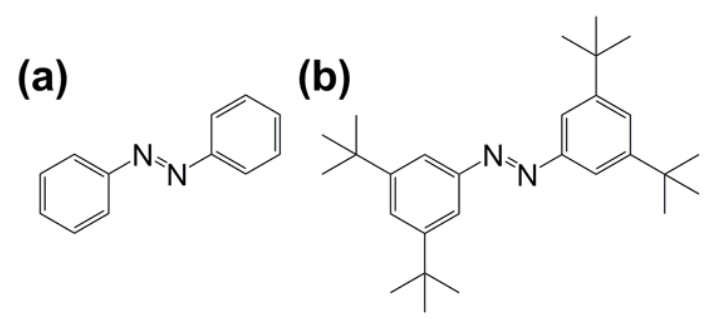

Figure 1: Molecular switches: (a) azobenzene, (b) 3,3',5,5'-tetra-tert-butyl-azobenzene (TBA).

of the entire molecule-substrate complex. Our argument is based on precise measurements of molecular adsorption geometries for azobenzene-based switches and corresponding large-scale density-functional theory (DFT) calculations.

If the observed restoration of the switching properties in the 3,3 ',5,5'tetra-tert-butyl-azobenzene (TBA) derivative on $\mathrm{Au}(111)$ was just a consequence of geometric decoupling, achieved through a modified molecular gasphase structure that remains undistorted on adsorption, one would expect this concept to work irrespective of the substrate material. However, even on the closely related $\operatorname{Ag}(111)$ surface, photoisomerization has neither been observed for azobenzene nor TBA [15]. To understand the reason behind this failure, we first analyze the detailed adsorption geometry of TBA on $\operatorname{Ag}(111)$ with the normal incidence X-ray standing wave technique (NIXSW) and compare it to the geometry of azobenzene $/ \operatorname{Ag}(111)$ that was reported earlier [16].

NIXSW allows model-free measurements of vertical adsorption heights above single crystalline surfaces, with an accuracy of about $0.05 \AA[17,18,19]$. It is thus an ideal tool to determine the vertical bonding distances between 
(a)
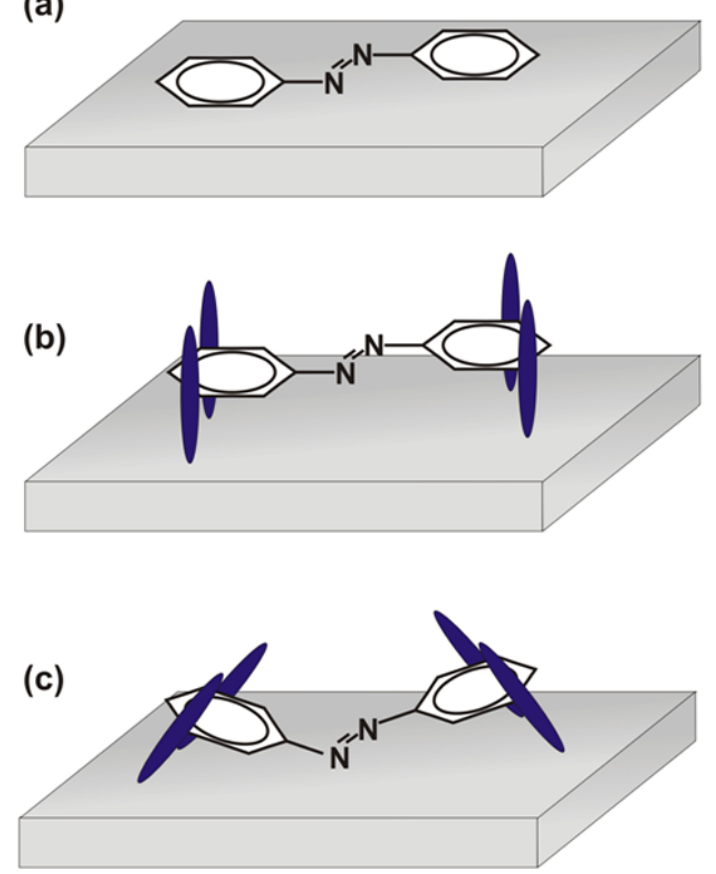

Figure 2: Schematic illustration of the geometric decoupling strategy and its failure in the context of the adsorbed azobenzene switch: (a) parent (unsubstituted) azobenzene, (b) expected lift-off by bulky spacer groups and (c) real adsorption geometry according to NIXSW and DFT. 
the functional $-\mathrm{N}=\mathrm{N}$ - unit in adsorbed azobenzene or TBA, thereby directly addressing the validity of the geometric decoupling concept displayed schematically in Fig. 2a/b. An illustration of the NIXSW experiment is shown in Fig. 3a. In the X-ray standing wave field, the photoelectron intensities from the various atoms of an adsorbed molecule depend on their respective distances to the Bragg planes of the substrate. Essentially, this height information can be separately retrieved for each chemical species from the dependence of the corresponding photoelectron yield on the energy of the incident X-ray beam $[17,18,19]$. This standing wave effect is illustrated in Fig. 3c-d for the N1s core level of nitrogen in TBA/Ag(111).

Analyzing the photoelectron yield for the diazo-group in TBA on $\mathrm{Ag}(111)$, we determine a nitrogen-silver adsorption distance $d_{N-A g}$ of $3.21 \pm 0.05 \AA$. Remarkably, this is only $0.14 \AA$ larger than the corresponding value for azobenzene $/ \operatorname{Ag}(111)(3.07 \AA)$ [16]. DFT calculations with a correction scheme to include dispersive van der Waals interactions [20, 21] quantitatively confirm this trend: For TBA $/ \operatorname{Ag}(111)$ we calculate $d_{N-A g}=3.11 \AA$, only $0.13 \AA$ larger than for azobenzene $\operatorname{Ag}(111)(2.98 \AA)$ [16]. If the tert-butyl groups in TBA indeed served as effective geometrical spacers, i.e. if the idea of a vertical lifting of the central flat backbone as sketched in Figs. 2a/b was correct, a much larger distance difference of $1.70 \AA$ would be expected from the respective gas-phase molecular geometries. The reason why this difference is much smaller on $\mathrm{Ag}(111)$ is that the real TBA adsorption geometry as determined by our DFT calculations is much better characterized as the "suspended bridge" between tilted tert-butyl legs that is shown in Fig. 2c.

While the experimental and theoretical analysis thus rationalizes a simi- 

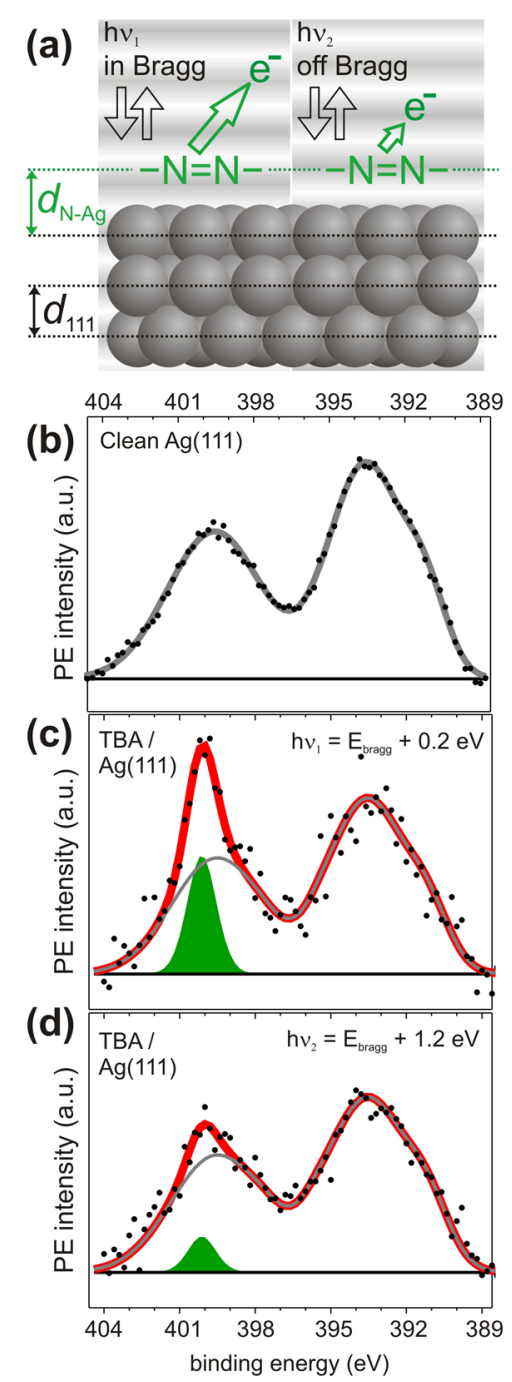

Figure 3: (a) Photoelectron emission enhanced (close to the Bragg condition, $\mathrm{E}_{\text {Bragg }}=2626.4 \mathrm{eV}$ ) or diminished (off Bragg) due to interference of incident and reflected X-ray beams, (b) experimental photoemission spectrum of clean $\mathrm{Ag}(111)$ in the energy range of Ag3d plasmons (dots) and corresponding fit (grey line); (c,d) normalized experimental photoemission spectra of $\mathrm{TBA} / \mathrm{Ag}(111)$ (dots) recorded at different incident beam energies ( $\mathrm{c}$ - in Bragg and d - off Bragg) in a single NIXSW experiment. Grey lines indicate the Ag3d plasmonic contribution according to (b), red lines are the fits of experimental data, and the green shaded areas represent the N1s core level peak. 
larly small switching probability for both TBA and azobenzene on $\mathrm{Ag}(111)$ - essentially because the spacer group concept fails - it still leaves the puzzle why TBA does switch on $\mathrm{Au}(111)$. To further elucidate this we are encouraged by the quantitative agreement between experiment and theory that was reached for the adsorption geometries at $\mathrm{Ag}(111)$. This indicates that in contrast to mere semi-local DFT [22] the here employed dispersion-corrected approach $[20,21]$ offers an accuracy with predictive quality for these systems. By carrying out corresponding calculations for TBA and azobenzene on $\mathrm{Au}(111)$, we arrive at the surprising finding that also here there is only an insignificant difference between the adsorption heights: $3.22 \AA$ for azobenzene versus $3.28 \AA$ for TBA. So, also on $\mathrm{Au}(111)$ the $-\mathrm{N}=\mathrm{N}$ - switching unit is not significantly lifted by the spacer groups.

These similarities in the adsorption geometries of azobenzene and TBA at $\mathrm{Ag}(111)$ and $\mathrm{Au}(111)$ largely disprove the purely geometrical arguments that are at the heart of the bulky spacer group concept. Instead, the present analysis supports the suggestion of Tegeder et al. [23] that for understanding the mechanism of the photo-induced switching functionality the detailed electronic structure of the metal substrate is much more important than previously thought. In particular, the different position of the $d$-band in $\mathrm{Ag}$ $(\approx-4 \mathrm{eV})$ and $\mathrm{Au}(\approx-2.1 \mathrm{eV})$ seems to be a key factor in determining the differences observed for these two substrates. In their model, Tegeder et al. explain the switching/non-switching of TBA on $\mathrm{Au}(111)$ and $\mathrm{Ag}(111)$, respectively, by assuming that the incident photon creates a hole in the metal, which rapidly relaxes to the upper edge of the $d$-band. In the case of $\mathrm{Au}(111)$, this hole can then efficiently transfer to the highest occupied molecular orbital 
(HOMO) of TBA, in contrast to $\mathrm{Ag}(111)$ where the lower lying $d$-band and correspondingly small overlap with the HOMO prevent this process. While the details of this model remain to be confirmed, it is clear that the existing data are difficult to reconcile without invoking new substrate-mediated isomerization mechanisms. This highlights the limitation of an approach to tune the switching functionality by engineering the distance between molecule and substrate alone and assuming the same switching mechanism at the surface than for the free molecule.

In conclusion, the present example of azobenzene-based molecular switches demonstrates that tuning the functionality of adsorbed molecules can only be successful if both the geometric and electronic structure of the complete molecule-substrate complex are taken into account. The physical mechanism governing the desired functionality may be influenced profoundly by the electronic interaction with the substrate. Therefore, in spite of their simplicity and common sense appeal, purely structural arguments to predict the functionality of adsorbed molecules have to be taken with much care, as they may turn out to be deceptive.

\section{Acknowledgment}

Funding by the DFG through SFB658 and TA244 and computing time at the Leibniz Supercomputing Centre are gratefully acknowledged. We thank J. Zegenhagen and Y. Mi (ESRF-Grenoble), O. Neucheva (FZJ-Jülich) for experimental support as well as M.V. Peters and S. Hecht (HU-Berlin) for the preparation of the azobenzene derivative.

\section{Appendix: Experimental and calculation details}

NIXSW experiments have been conducted at the beam line ID 32 of 
the European Synchrotron Radiation Facility in Grenoble under UHV conditions. The $\operatorname{Ag}(111)$ surface was cleaned in conventional way by several cycles of sputtering with $\mathrm{Ar}^{+}$ions and annealing at $820 \mathrm{~K}$. Azobenzene and TBA multilayers were deposited from a home-built evaporator $(385 \mathrm{~K}$, evaporation time $5 \mathrm{~min}$ ) onto the $\mathrm{Ag}$ crystal kept at $220 \mathrm{~K}$. Subsequent annealing (at $288 \mathrm{~K}$ for azobenzene [16], at $380 \mathrm{~K}$ for TBA) causes the desorption of multilayers, leaving behind a monolayer of the corresponding molecules on $\operatorname{Ag}(111)$. The desorption rate was preliminary calibrated and controlled in the final preparation by quadrupole mass spectrometer. In our NIXSW experiments the (111) Bragg reflection of Ag was exploited (azobenzene: $\mathrm{T}_{\text {Sample }}=130 \mathrm{~K}, \mathrm{E}_{\text {Bragg }}=2634.3 \mathrm{eV}$; TBA: $\mathrm{T}_{\text {Sample }}=300 \mathrm{~K}$, $\left.\mathrm{E}_{\text {Bragg }}=2626.4 \mathrm{eV}\right)$. Beam damage of the organic layer was excluded by $\mathrm{x}-$ ray photoelectron spectroscopy (XPS) before and after NIXSW experiments. Photoelectron yield curves (i.e. integrated XPS peak intensities as a function of photon energy) for the N1s core level were fitted with the program DARE (using non-dipolar parameters $\mathrm{Q}=0.22, \Delta=-0.26$ [24]), yielding two structural parameters, the coherent position $d_{c}$ and the coherent fraction $f_{c}$. The former is related to the average position of a certain species relative to the Bragg plane, e.g. $d_{N-A g}$ - the nitrogen-silver adsorption distance; the latter characterizes the statistical distribution of this position.

The DFT calculations were performed with a locally modified version of the CASTEP code [25], using the semi-local PBE functional to treat electronic exchange and correlation [26] and exactly the same computational setup as described in $[16,22]$. In brief, this setup comprises supercell geometries with seven layer inversion-symmetric slabs, $(6 \times 5)$ surface unit-cells and at 
least $18 \AA$ vacuum separation. Detailed convergence tests show that at the employed plane wave cutoff of $450 \mathrm{eV}$ and $(2 \times 3 \times 1)$ Monkhorst-Pack (MP) grid the reported structural parameters are numerically converged to $0.01 \AA$.

In the spirit of the DFT-D approach, the lack of vdW interactions in the employed semi-local functional is approximately corrected with an additional analytical, two-body inter-atomic potential. At long range, this potential equals the leading $C_{6} R^{-6}$-term of the London series, where $R$ is the interatomic distance, and $C_{6}$ - the so-called dispersion coefficient. At short range, this long range potential is matched to the DFT inter-atomic potential by a damping function $f\left(R, R^{0}\right)$, typically modulated by the vdW radii $R^{0}$ of the atom pair. In this work, we use the material-specific $C_{6}$ and $R^{0}$ parameters, as well as the damping function form suggested by Tkatchenko and Scheffler [20].

\section{References}

[1] T. A. Jung, R. R. Schlittler, J. K. Gimzewski, Nature 386 (1997) 696.

[2] F. Moresco, G. Meyer, K.-H. Rieder, H. Tang, A. Gourdon, C. Joachim, Phys. Rev. Lett. 86 (2001) 672.

[3] M. J. Comstock, N. Levy, A. Kirakosian, J. Cho, F. Lauterwasser, J. H. Harvey, D. A. Strubbe, J. M. J. Fréchet, D. Trauner, S. G. Louie, M. F. Crommie, Phys. Rev. Lett. 99 (2007) 038301.

[4] M. Alemani, M. V. Peters, S. Hecht, K.-H. Rieder, F. Moresco, L. Grill, J. Am. Chem. Soc. 128 (2006) 14446.

[5] S. Hagen, F. Leyssner, D. Nandi, M. Wolf, P. Tegeder, Chem. Phys. Lett. 444 (2007) 85. 
[6] L. Óvári, M. Wolf, P. Tegeder, J. Phys. Chem. C 111 (2007) 15370.

[7] S. Hagen, P. Kate, M. V. Peters, S. Hecht, M. Wolf, P. Tegeder, Appl. Phys. A 93 (2008) 253.

[8] M. Wolf, P. Tegeder, Surf. Sci. 603 (2009) 1506.

[9] H. Rau, in Photochromism-Molecules and Systems, (Eds: H. Dürr, H. Bouas-Laurent), Elsevier, Amsterdam, 2003, p. 165.

[10] C. Gahl, K. Ishioka, Q. Zhong, A. Hotzel, M. Wolf, Faraday Discuss. 117 (2000) 191.

[11] H. Petek, H. Nagano, M. J. Weida, S. Ogawa, J. Phys. Chem. B 105 (2001) 6767.

[12] P. S. Kirchmann, P. A. Loukakos, U. Bovensiepen, M. Wolf, New J. Phys. 7 (2005) 113.

[13] G. Dutton, D. P. Quinn, C. D. Lindstrom, X.-Y. Zhu, Phys. Rev. B 72 (2005) 045441.

[14] A. Yang, S. T. Shipman, S. Garrett-Roe, J. Johns, M. Strader, P. Szymanski, E. Müller, C. Harris, J. Phys. Chem. C 112 (2008) 2506.

[15] P. Tegeder, S. Hagen, F. Leyssner, M. V. Peters, S. Hecht, T. Klamroth, P. Saalfrank, M. Wolf, Appl. Phys. A 88 (2007) 465.

[16] G. Mercurio, E. R. McNellis, I. Martin, S. Hagen, F. Leyssner, S. Soubatch, J. Meyer, M. Wolf, P. Tegeder, F. S. Tautz, K. Reuter, Phys. Rev. Lett. 104 (2010) 036102. 
[17] J. Zegenhagen, Surf. Sci. Rep. 18 (1993) 199.

[18] D. P. Woodruff, Prog. Surf. Sci. 57 (1998) 1.

[19] D. P. Woodruff, Prog Phys. 68 (2005) 743.

[20] A. Tkatchenko, M. Scheffler, Phys. Rev. Lett. 102 (2009) 073005.

[21] E. R. McNellis, J. Meyer, K. Reuter, Phys. Rev. B 80 (2009) 205414.

[22] E. McNellis, J. Meyer, A. Dehghan-Baghi, K. Reuter, Phys. Rev. B 80 (2009) 035414.

[23] S. Hagen, P. Kate, F. Leyssner, D. Nandi, M. Wolf, P. Tegeder, J. Chem. Phys. 129 (2008) 164102.

[24] C. Stadler, S. Hansen, F. Pollinger, C. Kumpf, E. Umbach, T.-L. Lee and J. Zegenhagen, Phys. Rev. B 74 (2006) 035404.

[25] S. J. Clark, M. D. Segall, C. J. Pickard, P. J. Hasnip, M. I. J. Probert, K. Refson and M. C. Payne, Z. Kristallogr. 220 (2005) 567.

[26] J. P. Perdew, K. Burke, M. Ernzerhof, Phys. Rev. Lett. 77 (1996) 3865. 